\title{
A Video Watermarking Procedure Based on XML Documents
}

\author{
Franco Frattolillo and Salvatore D’Onofrio \\ Research Centre on Software Technology, \\ Department of Engineering, University of Sannio, Italy \\ frattolillo@unisannio.it
}

\begin{abstract}
This paper presents a watermarking procedure for MPEG-2 videos based on the use of XML documents. The procedure enables the copyright owner to insert a distinct watermark identifying the buyer within the distributed videos. To increase the security level of the procedure, the watermark is repeatedly embedded into some selected I-frames in the DCT domain at different frequencies and by exploiting both block classification techniques and perceptual analysis. The embedded watermark is then extracted from a video according to the information contained in a protected XML document associated to the video.
\end{abstract}

\section{Introduction and Motivations}

Digital watermarking [1] can be considered a main technology to implement the copyright protection of digital contents distributed on the Internet. To this end, many recent watermarking procedures achieve high levels of security and robustness by adopting "readable" watermarking schemes based on "blind" and not publicly available decoders as well as letting the inserted watermarks depend on the host signals 2. However, the "nonblind" watermarking schemes are typically considered more robust then the blind ones 3 3.4. Unfortunately, differently from the blind ones, the nonblind schemes need the original contents to run the watermark detection or extraction algorithms. This is considered a relevant drawback particularly for the procedures that aim at being adopted in a web context, because such procedures force the distinct web entities involved in "identification and arbitration" protocols [4 to exchange and store the original unprotected, large size digital contents, thus risking "colluding" behaviors [234/5].

This paper presents a watermarking procedure for MPEG-2 compressed videos based on the use of XML documents. The procedure enables the copyright owner to insert a distinct code identifying the buyer within each copy of the distributed videos. Furthermore, to increase the security level of the procedure, the watermark is repeatedly embedded into some selected I-frames of a video in the DCT domain at different frequencies and by exploiting both block classification techniques and perceptual analysis. The embedded watermark is then extracted from a video according to the information contained in a protected XML document associated to the video. Thus, the usual security and robustness 
levels characterizing the nonblind watermarking schemes can be achieved without requiring the original unprotected, large size videos to be exchanged in the Internet whenever the watermark extraction has to be performed. Furthermore, using the XML technology makes it also easier to automate the document access in a web context, since XML is a technology well supported by the Java world, and document parsers, such as SAX and DOM parsers, are freely available.

The paper is organized as the follows. Section 2 describes the proposed watermarking procedure. Section 3 describes the watermark extraction procedure. Section 4 reports on some preliminary experimental results. Section 5 reports conclusion remarks.

\section{The Watermarking Procedure}

The watermarking procedure makes it possible to insert into an MPEG-2 video a binary code represented by a sequence of bits $\mu \in\{0,1\}$ and able to unambiguously identify a user. The sequence $\mu$, whose length is denoted as $n_{\mu}$, is repeatedly embedded into each of some selected I-frames of the video, denoted as $\epsilon_{1}, \epsilon_{2} \ldots \epsilon_{r}$, in the DCT domain at different frequencies, denoted as $\gamma_{1}, \gamma_{2} \ldots$ $\gamma_{f}$. In particular, since the coefficients in each $8 \times 8$ DCT block have a frequency value associated with them, a $\gamma$ value identifies an entry in such blocks, and so, it can range from 1 to $8^{2}=64$. Furthermore, to increase the security level of the procedure, the watermark insertion is assumed to be usually carried out at low, middle and high frequencies chosen on the basis of the video to watermark.

In principle, all the DCT coefficients of an I-frame could be modified by a value representing a watermark information. However, in the proposed procedure, the "perceptual capacity" of the coefficients belonging to the luminance DCT blocks of the selected I-frames is preliminarily estimated by exploiting both block classification techniques and perceptual analysis. In fact, the block classification techniques [67] are applied to indicate the bests DCT coefficients of the selected I-frames that can be altered without reducing the visual quality. They classify each luminance DCT block with respect to its energy distribution. The result of this procedure is a first selection of DCT coefficients whose modification has a minimal or no impact to the perceptual quality of the selected I-frames.

The perceptual analysis is then applied to calculate the "just noticeable difference" $(j n d)$ values for the DCT coefficients [8|9]10. Such values are the thresholds beyond which any changes to the respective coefficient will most likely be visible. Therefore, let $X_{b_{m}}^{\epsilon}(\gamma)$ denote the DCT coefficient at the frequency $\gamma$ in the block $b_{m}$ of the I-frame $\epsilon$, and let $J N D_{b_{m}}^{\epsilon}(\gamma)$ denote the $j n d$ value calculated for the $X_{b_{m}}^{\epsilon}(\gamma)$ coefficient. $J N D_{b_{m}}^{\epsilon}(\gamma)$ can be approximated by the following expression:

$$
J N D_{b_{m}}^{\epsilon}(\gamma) \approx \max \left\{C_{b_{m}}^{\epsilon}(\gamma),\left|C_{b_{m}}^{\epsilon}(\gamma)\right| E_{b_{m}}^{\epsilon}(\gamma)^{g}\right\}
$$

where $C_{b_{m}}^{\epsilon}(\gamma)$ represents the perceptual threshold of the contrast masking and is expressed as:

$$
C_{b_{m}}^{\epsilon}(\gamma)=\max \left\{t_{b_{m}}^{\epsilon}(\gamma),\left|X_{b_{m}}^{\epsilon}(\gamma)\right|^{h} t_{b_{m}}^{\epsilon}(\gamma)^{1-h}\right\}
$$


$E_{b_{m}}^{\epsilon}(\gamma)$ is the entropy value calculated over the eight neighbors of the $X_{b_{m}}^{\epsilon}(\gamma)$ coefficient [8, and can be approximated by the following expression:

$$
E_{b_{m}}^{\epsilon}(\gamma) \approx X_{b_{m}}^{\epsilon}(\gamma)-u_{b_{m}}^{\epsilon}(\gamma) q(\gamma)
$$

In (11) $g$ is assumed equal to 0.5, while in (2) $h$ is assumed equal to 0.7 and $t_{b_{m}}^{\epsilon}(\gamma)$ is equal to $t(\gamma)\left(X_{b_{m}}^{\epsilon}(1) / X(1)\right)$, where $X(1)$ is a DC coefficient corresponding to the mean luminance of the display, while $X_{b_{m}}^{\epsilon}(1)$ is the DC coefficient of the block $b_{m}$ of the I-frame $\epsilon$. In fact, $t(\gamma)$ can be approximated by the value $q(\gamma) / 2$, where $q(\gamma)$ represents the coefficient of the quantization matrix corresponding to the frequency $\gamma\left[9\right.$. Finally, in (3) $u_{b_{m}}^{\epsilon}(\gamma)$ is equal to $\operatorname{round}\left(X_{b_{m}}^{\epsilon}(\gamma) / q(\gamma)\right)$.

The insertion procedure at the frequency $\gamma$ of a selected I-frame assumes that each bit of the sequence $\mu$ is inserted into the I-frame by altering a pair of DCT coefficients associated to the frequency $\gamma$ and chosen among the ones previously selected by applying the block classification techniques and perceptual analysis. In particular, the "choice rule" states that two DCT coefficients of a selected I-frame are allowed to belong to a same pair only if they have a similar value. Furthermore, to insert the bits of $\mu$ into an I-frame $\epsilon$, the "encoding function" $K$ has to be defined. $K$ defines an encoding rule by which the bits 0 and 1 are translated to the symbols of the alphabet composed by $\{\nearrow, \searrow\}$, respectively called the up symbol and the down symbol. Thus, a sequence $\mu \in$ $\{0,1\}$ is translated to a corresponding sequence $\sigma \in\{\nearrow, \searrow\}$ depending on the function $K$. For example, the sequence $\{01101 \ldots\}$ is translated to the sequence $\{\nearrow \searrow \searrow \nearrow \searrow \ldots\}$, if $K$ associates the $u p$ symbol to 0 and the down symbol to 1 .

Let $\mu$ be a user sequence, and let $\sigma$ be the sequence obtained by applying a $K$ function. Let $\gamma_{i}, \forall i=1 \ldots f$, be the insertion frequencies, and let $\epsilon_{p}, \forall p=1 \ldots r$, be the selected I-frames of the video. Let $W_{b_{m}}^{\epsilon_{p}}\left(\gamma_{i}\right)$ denote the watermarked DCT coefficient at the frequency $\gamma_{i}$ in the block $b_{m}$ of the I-frame $\epsilon_{p}$. A symbol of $\sigma$ is inserted into a pair of DCT coefficients belonging to the blocks $b_{m}$ and $b_{n}$, at the frequency $\gamma_{i}$ of the I-frame $\epsilon_{p}$, by the following expressions:

$$
\begin{gathered}
\left\{\begin{array}{l}
W_{b_{m}}^{\epsilon_{p}}\left(\gamma_{i}\right)=X_{b_{m}}^{\epsilon_{p}}\left(\gamma_{i}\right)-J N D_{b_{m}}^{\epsilon_{p}}\left(\gamma_{i}\right) \\
W_{b_{n}}^{\epsilon_{p}}\left(\gamma_{i}\right)=X_{b_{n}}^{\epsilon_{p}}\left(\gamma_{i}\right)+J N D_{b_{n}}^{\epsilon_{p}}\left(\gamma_{i}\right)
\end{array}\right. \text { to insert } \\
\left\{\begin{array}{l}
W_{b_{m}}^{\epsilon_{p}}\left(\gamma_{i}\right)=X_{b_{m}}^{\epsilon_{p}}\left(\gamma_{i}\right)+J N D_{b_{m}}^{\epsilon_{p}}\left(\gamma_{i}\right) \\
W_{b_{n}}^{\epsilon_{p}}\left(\gamma_{i}\right)=X_{b_{n}}^{\epsilon_{p}}\left(\gamma_{i}\right)-J N D_{b_{n}}^{\epsilon_{p}}\left(\gamma_{i}\right)
\end{array} \text { to insert } \searrow\right. \\
\forall i=1 \ldots f \text { and } \forall p=1 \ldots r
\end{gathered}
$$

In fact, as specified above, since the choice rule requires that $X_{b_{m}}^{\epsilon_{p}}\left(\gamma_{i}\right) \approx X_{b_{n}}^{\epsilon_{p}}\left(\gamma_{i}\right)$, $\forall i=1 \ldots f$ and $\forall p=1 \ldots r$, the insertion process ends up maximizing the difference existing between the coefficients of the pair according to the direction specified by the insertion symbol and by an amount that cannot compromise the final visual quality of the video. Consequently, the insertion process should be carried out according to the following rules:

1. The I-frames to watermark should qualify significant scenes of the video, and more than three consecutive I-frames should belong to each selected scene. 
2. The insertion frequencies should be evenly distributed among the low, middle and high frequencies, and should be chosen so that possible attacks characterized by a filtering behavior on the video frames would end up reducing the final video quality. This can be achieved by selecting the frequencies characterized by high spectrum values, which, if filtered, can impair the video.

3. At each insertion frequency and for each selected I-frame, the pairs of the selected DCT coefficients should belong to spatial regions that cannot be cropped without impairing the video.

Once the symbols of the sequence $\sigma$ have been inserted into the selected I-frames at the chosen frequencies, in order to increase the security level of the watermarking procedure against "collusion" attacks 3.5, it is necessary to hide the modifications made to the DCT coefficients of the I-frames. In fact, let $\gamma_{1}, \gamma_{2} \ldots \gamma_{f}$ be the insertion frequencies chosen for the video, and let $\Sigma^{\epsilon_{p}}\left(\gamma_{i}\right)$ denote the sequences of the pairs of DCT entries $\left(b_{m}^{\epsilon_{p}}\left(\gamma_{i}\right), b_{n}^{\epsilon_{p}}\left(\gamma_{i}\right)\right)$ that have been involved in the watermarking process for a given frequency $\gamma_{i}, \forall i=1 \ldots f$ and for a given I-frame $\epsilon_{p}, \forall p=1 \ldots r$. It is worth noting that both the set of the frequencies $\gamma_{i}$ and the sets $\Sigma^{\epsilon_{p}}\left(\gamma_{i}\right)$ are always the same for all the copies of a given video to protect. Consequently, the DCT coefficients modified at the different insertion frequencies in the selected I-frames remain the same for all the copies of the video. Therefore, in order to prevent malicious users from individuating the DCT coefficients modified by the insertion process, the procedure adds the $j n d$ values modulated by a binary pseudo-noise sequence $\rho \in\{-1,1\}$ to all the unmodified DCT coefficients of the selected I-frames of the watermarked video:

$$
\begin{gathered}
X_{b_{k}}^{\epsilon_{p}}\left(\gamma_{i}\right)=X_{b_{k}}^{\epsilon_{p}}\left(\gamma_{i}\right)+\alpha_{k} \rho_{k} J N D_{b_{k}}^{\epsilon_{p}}\left(\gamma_{i}\right), \\
(\forall p=1 \ldots r) \text { and }\left((i \neq 1 \ldots f) \text { or }\left(i=1 \ldots f \text { and } b_{k} \notin \Sigma^{\epsilon_{p}}\left(\gamma_{i}\right)\right)\right)
\end{gathered}
$$

where $0<\alpha_{k}<0.5$ is a randomly varied amplitude factor.

\subsection{The XML Documents}

The capability of both repeatedly embedding a user code at different frequencies and hiding the watermarked DCT coefficients in each of the selected I-frames of the video can make the proposed procedure almost secure against the most common filtering, corrupting, removal, averaging and collusion attacks. However, the characteristics of the insertion process could make the procedure vulnerable to geometric attacks. Therefore, to increase the robustness level of the procedure against such attacks, the attacked videos should be geometrically re-synchronized before carrying out the watermark extraction.

To detect the most common geometric distortions applied to a watermarked video without having to use complex re-synchronization techniques, the proposed procedure makes use of some information about the video, which is assumed to be stored in a protected XML document associated to it. This allows the information to be stored in both textual and quantitative form. The textual information can individuate and describe the scenes which the I-frames belong to, and some evident and significant "feature points" and boundary segments of the selected 
I-frames. The quantitative information can provide the original dimensions of the video, the coordinates of the feature points and selected boundaries, some Fourier descriptors and statistical moments of $K$-point digital boundaries, as well as the eigenvectors and eigenvalues of some well-defined regions of the selected I-frames. Thus, inverse geometric transformations can be performed on the attacked video in order to restore it before the watermark extraction [1].

Therefore, the XML document associated to each video to protect has to include: (1) the insertion frequencies $\gamma_{1}, \gamma_{2} \ldots \gamma_{f} ;(2)$ the selected I-frames $\epsilon_{1}, \epsilon_{2} \ldots \epsilon_{r}$, also specified in terms of significant scenes which they refer to; (3) the sets $\Sigma^{\epsilon_{p}}\left(\gamma_{i}\right), \forall i=1 \ldots f$ and $\forall p=1 \ldots r$; (4) the definition of the encoding function $K$; (5) some further information about the original video, which can synthetically characterize the video and can be exploited to individuate possible geometric modifications performed on it.

\section{The Watermark Extraction}

The first operation to perform before carrying out the watermark extraction from a protected video is its geometric re-synchronization. Therefore, let Figure 1(a) be an I-frame of the "Cactus" video. Let Figures 1(b) and 1(d) be respectively the corresponding watermarked and attacked version. The geometric re-synchronization can be carried out by exploiting the information stored in the XML document associated to the "Cactus" video in order to build a reference picture (Figure 1(e)) whose dimensions coincide with the ones of the original I-frame. Then, the feature points connected by segments and specified by the XML document are to be reported on this picture. These points have been originally determined on the watermarked I-frame (Figure 1(c)) and are: the two upper edges of the glass, whose coordinates are $\left(x_{1}, y_{1}\right)$ and $\left(x_{2}, y_{2}\right)$; the upper left edge of the comb, specified by $\left(x_{3}, y_{3}\right)$; the tip of the nose represented on the glass, specified by $\left(x_{4}, y_{4}\right)$. The coordinates are referred to the $X$ and $Y$ axis, and the dimensions of the original I-frame are respectively $d_{x}$ and $d_{y}$.

The successive operation consists in reporting the feature points connected by segments on the attacked version of the I-frame (Figure 1(d)). In particular, the attacked I-frame is a scaled and $45^{\circ}$ rotated version of the watermarked I-frame (Figure 1(b)). To this end, it is worth noting that the feature points can be reported on the I-frame solely starting from the textual description provided by the XML document associated to the video (Figures 1(d) and 1(f)). This entails a natural approximation in individuating the feature points on the attacked I-frame, which can determine errors in the geometric re-synchronization process. However, the preliminary tests, conducted also on other videos, have shown that the proposed procedure is robust with respect to such approximations. In fact, the procedure has been able to ensure a correct watermark extraction provided that the rotation degrees and scale factors are determined with approximations in the range about $\pm 6 \%$. However, these limits have never been exceeded in the conducted, practical tests. To this end, Figure 2 shows the result of the worst re-synchronization performed on the scaled and $45^{\circ}$ rotated version of 


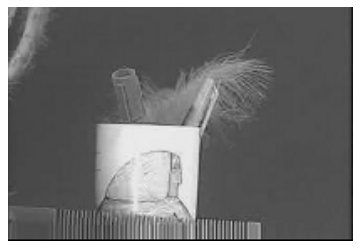

(a)

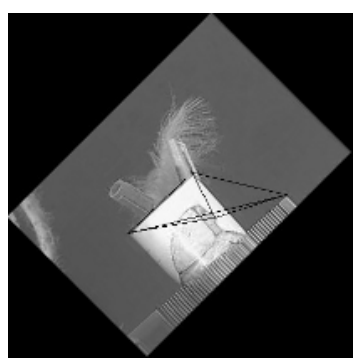

(d)

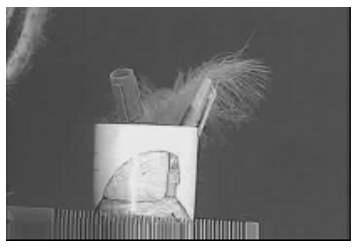

(b)

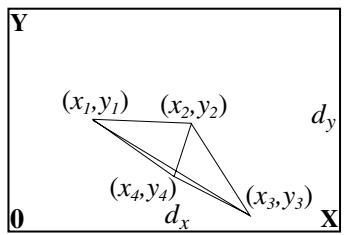

(e)

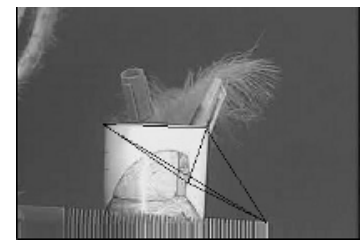

(c)

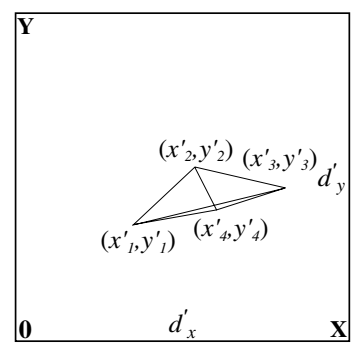

(f)

Fig. 1. The process of geometric re-synchronization of a "Cactus" I-frame
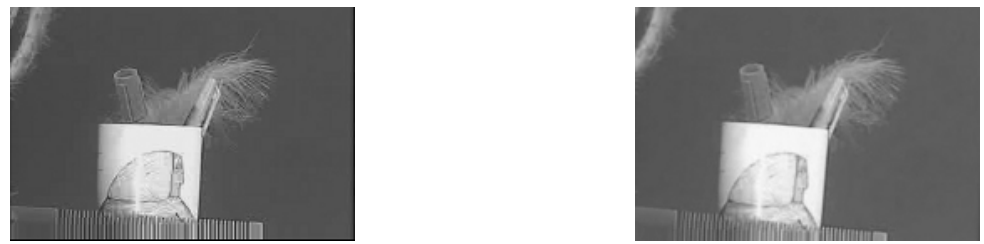

Fig. 2. A "Cactus" watermarked I-frame and its re-synchronized version

the watermarked I-frame, which has not anyway prevented a correct watermark extraction, as reported in Section 4. In fact, the imperfect re-building of the I-frame essentially affects the outer regions of the image, i.e. the regions that do not, and should not, host watermark information.

After the geometric re-synchronization, the watermark extraction can be carried out. In particular, for each insertion frequency $\gamma_{i}$ and for each selected I-frames $\epsilon_{p}$, the pairs of coefficients specified by the DCT entries $\left(b_{m}^{\epsilon_{p}}\left(\gamma_{i}\right), b_{n}^{\epsilon_{p}}\left(\gamma_{i}\right)\right)$ belonging to the set $\Sigma^{\epsilon_{p}}\left(\gamma_{i}\right)$, with $i=1 \ldots f$ and $p=1 \ldots r$, have to be examined. Therefore, let $\hat{W}_{b_{m}}^{\epsilon_{p}}\left(\gamma_{i}\right)$ and $\hat{W}_{b_{n}}^{\epsilon_{p}}\left(\gamma_{i}\right)$ be the two coefficients of a pair belonging to $\Sigma^{\epsilon_{p}}\left(\gamma_{i}\right)$. To extract the watermark symbol they host, the following expression has to be calculated:

$$
\left\{\begin{array}{l}
\hat{W}_{b_{m}}^{\epsilon_{p}}\left(\gamma_{i}\right)-\hat{W}_{b_{n}}^{\epsilon_{p}}\left(\gamma_{i}\right)>0 \Longrightarrow \searrow \text { is extracted } \\
\hat{W}_{b_{m}}^{\epsilon_{p}}\left(\gamma_{i}\right)-\hat{W}_{b_{n}}^{\epsilon_{p}}\left(\gamma_{i}\right)<0 \Longrightarrow \nearrow \text { is extracted }
\end{array}\right.
$$

Then, the extracted symbol is translated to a bit depending on the $K$ function.

After having completed the watermark extraction process, $f \cdot r$ user sequences $\mu_{i, p}$ result in being re-built, one for each different insertion frequency $\gamma_{i}$ and 
Table A

\begin{tabular}{|c|c|c|c|}
\hline Attack & $\boldsymbol{f}$ & $\boldsymbol{r}$ & ber (\%) \\
\hline Add Noise & 3 & 9 & 2.03 \\
Add Noise & 6 & 12 & 1.97 \\
Add Noise & 9 & 15 & 1.85 \\
Sharpening & 3 & 9 & 1.64 \\
Sharpening & 6 & 12 & 1.56 \\
Sharpening & 9 & 15 & 1.48 \\
Median & 3 & 9 & 1.89 \\
Median & 6 & 12 & 1.62 \\
Median & 9 & 15 & 1.53 \\
Rotating $45^{\circ}$ & 3 & 9 & 3.12 \\
Rotating $45^{\circ}$ & 6 & 12 & 2.98 \\
Rotating $45^{\circ}$ & 9 & 15 & 2.61 \\
\hline \multicolumn{4}{|r}{}
\end{tabular}

Table B

$\left.\begin{array}{|c|c|c|c|c|}\hline \text { Table B } \\ \hline \text { Attack } & \boldsymbol{f} & \boldsymbol{r} & \boldsymbol{k} & \text { ber }(\%) \\ \hline \text { Averaging } & 3 & 9 & 5 & 5.21 \\ \text { Averaging } & 6 & 12 & 10 & 6.05 \\ \text { Minimum } & 3 & 9 & 5 & 3.46 \\ \text { Minimum } & 6 & 12 & 10 & 3.91 \\ \text { Minimum } & 9 & 15 & 15 & 4.38 \\ \text { Median } & 3 & 9 & 5 & 4.16 \\ \text { Median } & 6 & 12 & 10 & 4.93 \\ \text { Median } & 9 & 15 & 15 & 5.67 \\ \text { Maximum } & 3 & 9 & 5 & 3.52 \\ \text { Maximum } & 6 & 12 & 10 & 3.99 \\ \text { Mallusion } \\ \text { attacks } \\ \\ \text { Maximum } & 9 & 15 & 15 & 4.37\end{array}\right\} \begin{gathered}\text { Nollusion } \\ \text { attacks } \\ \text { linear } \\ \text { collang } \\ \end{gathered}$

Fig. 3. The results of some preliminary tests

I-frame $\epsilon_{p}$. Therefore, let $\mu(j)$ denote the $j$-th bit in the user sequence $\mu . \mu(j)$ can be derived from the sequences $\hat{\mu_{i, p}}$ by the following expression:

$$
\mu(j) \equiv 1 \Longleftrightarrow \frac{\sum_{i=1}^{f} \sum_{p=1}^{r} \mu_{i, p}(j)}{f \cdot r}>0.5, \quad \forall j=1 \ldots n_{\mu}
$$

\section{Experimental Results}

The robustness and security of the proposed procedure have been assessed by measuring the bit error rate (ber) under a different number of the insertion frequencies $f$ and of the watermarked I-frames $r$. The ber is expressed in percentage and is calculated as $\left(\sum_{i=1}^{f} \sum_{p=1}^{r} b_{e r} r_{i, p}\right) / f \cdot r$, where $b e r_{i, p}$ is the number of bit errors reported in the watermark extraction carried out at the frequency $\gamma_{i}$ from the I-frame $\epsilon_{p}$. The user sequence $\mu$ is assumed 128 bit long, while the video used to perform the tests is the "Cactus" video and is coded at $30 \mathrm{fps}$, with a resolution of $328 \times 224$ pixels and a duration about 120 seconds. However, further tests have been conducted on other videos freely available on the Internet, but the obtained results have not been reported for the sake of brevity.

Table A in Figure 3 summarizes the results obtained under "simple" or "detection-disabling" attacks. In fact, the "simple" attacks attempt to impair the embedded watermark by manipulations of the whole watermarked video, such as filtering or compression manipulations or addition of noise. The "detectiondisabling" attacks attempt to break the correlation and to make the recovery of the watermark impossible or infeasible, mostly by geometric distortion like rotation. Table B in Figure 3 shows the results obtained under some "linear" and "non linear" collusion attacks, whose correct definitions are reported in [5].In particular, $k$ denotes the number of the colluding videos, while the anticollusion codes used in the tests have been generated according to what reported in [1].

The results reported in Figure 3 show that the proposed procedure can achieve a good performance against attacks that are considered able to prove a high level of robustness and security. In particular, the ber values are always low, 
and the user sequence $\mu$ has been always correctly re-built from the sequences extracted for each test by applying the expression (4). In fact, the redundancy assured by the insertion process enables the procedure to behave as other well known, robust and secure watermarking procedures. This also because the procedure allows for choosing the insertion frequencies as well as the regions of the selected I-frames where to embed the watermark information. Moreover, in the conducted tests, the values of $f$ and $r$ are rather small, but they can be also increased in order to improve the security and robustness levels of the procedure without compromising the final visual quality of the video.

\section{Conclusions}

In this paper a watermarking procedure for the copyright protection of MPEG-2 videos is presented. The procedure directly acts on compressed videos and exploits protected XML documents to store information needed to the watermark extraction. The redundancy assured by the insertion process at different frequencies and for each of the selected I-frames enables the procedure to achieve a good performance against the most common and dangerous attacks. In addition, the procedure can also improve its security and robustness levels by increasing the number of the insertion frequencies and of the watermarked I-frames.

\section{References}

1. Cox, I., Bloom, J., Miller, M.: Digital Watermarking: Principles \& Practice. Morgan Kaufman (2001)

2. Barni, M., Bartolini, F.: Data hiding for fighting piracy. IEEE Signal Processing Magazine 21 (2004) 28-39

3. Trappe, W., Wu, M., et al.: Anti-collusion fingerprinting for multimedia. IEEE Trans. on Signal Processing 41 (2003) 1069-1087

4. Lei, C.L., Yu, P.L., et al.: An efficient and anonymous buyer-seller watermarking protocol. IEEE Trans. on Image Proces. 13 (2004) 1618-1626

5. Wu, M., Trappe, W., et al.: Collusion-resistant fingerprinting for multimedia. IEEE Signal Processing Magazine 21 (2004) 15-27

6. Chung, T.Y., Hong, M.S., et al.: Digital watermarking for copyright protection of MPEG2 compressed video. IEEE Trans. on Consumer Electr. 44 (1998) 895-901

7. Wang, Y., Ostermann, J., Zhang, Y.: Video Processing and Communications. Prentice Hall (2002)

8. Kim, S.W., Suthaharan, S.: An entropy masking model for multimedia content watermarking. In: Procs of the 37th Hawaii Intl Conference on System Sciences, IEEE Computer Society (2004) 70182.2

9. Watson, A.B.: DCT quantization matrices visually optimized for individual images. In Allebach, J.P., Rogowitz, B.E., eds.: Human Vision, Visual Processing and Digital Display IV. Volume 1913 of SPIE Procs., S. Jose, CA, USA (1993) 202-216

10. Wolfgang, R.B., et al.: Perceptual watermarks for digital images and video. Procs of the IEEE 87 (1999) 1108-1126

11. Boneh, D., Shaw, J.: Collusion-secure fingerprinting for digital data. IEEE Trans. on Infor. Theory 44 (1998) 1897-1905 